\title{
Systemic therapy for metastatic renal cell carcinoma in the first-line setting: a systematic review and network meta-analysis
}

\author{
Keiichiro Mori ${ }^{1,2} \oplus$. Hadi Mostafaei ${ }^{1,3} \cdot$ Noriyoshi Miura $^{1,4} \cdot$ Pierre I. Karakiewicz ${ }^{5}$ Stefano Luzzago ${ }^{5,6} \cdot$ \\ Manuela Schmidinger ${ }^{7}$. Andreas Bruchbacher ${ }^{1,7} \cdot$ Benjamin Pradere $^{1,8,16} \cdot$ Shin Egawa $^{2}$. \\ Shahrokh F. Shariat ${ }^{1,9,10,11,12,13,14,15}$
}

Received: 8 June 2020 / Accepted: 23 July 2020 / Published online: 5 August 2020

(c) The Author(s) 2020

\begin{abstract}
Purpose Management of metastatic renal cell cancer (mRCC) has undergone a paradigm shift with immune-checkpoint inhibitors (ICI) in the first-line setting. However, direct comparative data are inadequate to inform treatment decisions. Therefore, we aimed to assess first-line therapy for $\mathrm{mRCC}$ and indirectly compare the efficacy and safety of currently available treatments.

Materials and methods Multiple databases were searched for articles published before June 2020. Studies that compared overall and/or progression-free survival (OS/PFS) and/or adverse events (AEs) in mRCC patients were considered eligible. Results Six studies matched our eligibility criteria. For OS, pembrolizumab plus axitinib [hazard ratio (HR) $0.85,95 \%$ credible interval (CrI) 0.73-0.98] and nivolumab plus ipilimumab (HR 0.86, 95\% CrI 0.75-0.99) were significantly more effective than sunitinib, and pembrolizumab plus axitinib was probably the best option based on analysis of the treatment ranking. For PFS, pembrolizumab plus axitinib (HR 0.86, 95\% CrI 0.76-0.97) and avelumab plus axitinib (HR 0.85, 95\% CrI 0.74-0.98) were statistically superior to sunitinib, and avelumab plus axitinib was likely to be the preferred option based on analysis of the treatment ranking, closely followed by pembrolizumab plus axitinib. Nivolumab plus ipilimumab had significantly lower rates of serious AEs than sunitinib.

Conclusion Pembrolizumab plus axitinib seemed to be the most efficacious first-line agents, while nivolumab plus ipilimumab had the most favorable efficacy-tolerability equilibrium. These findings may facilitate individualized treatment strategies and inform future direct comparative trials in an expanding treatment options without direct comparison between approved drugs.
\end{abstract}

Keywords Renal cell carcinoma $\cdot$ Network meta-analysis $\cdot$ First-line $\cdot$ Immune-checkpoint inhibitors

\section{Introduction}

Renal cell carcinoma (RCC) is among the top 10 most frequently diagnosed cancers worldwide [1]. Approximately $25 \%$ of patients with RCC who present with metastatic tumors at the time of initial diagnosis typically require systemic treatment. Moreover, another $20-50 \%$ of RCC patients with localized disease eventually develop metastatic RCC (mRCC) [2-4]. Targeted therapies with lesser toxicity and

Electronic supplementary material The online version of this article (https://doi.org/10.1007/s00262-020-02684-8) contains supplementary material, which is available to authorized users.

Shahrokh F. Shariat

shahrokh.shariat@meduriwien.ac.at

Extended author information available on the last page of the article higher survival benefits have become the mainstay of treatment for $\mathrm{mRCC}$ and multiple targeted therapies, such as tyrosine kinase inhibitors (TKI), mammalian target of rapamycin pathway inhibitors, and vascular endothelial growth factor (VEGF) monoclonal antibody, have been approved as first-line systemic treatments for mRCC [4-6]. More recently, immune-checkpoint inhibitors (ICI) have been provided as a further therapeutic option.

The selection of an appropriate first-line treatment is absolutely crucial, especially because data from the targeted therapy era suggest that only $50 \%$ of patients receive second-line treatment, and that only $20 \%$ receive thirdline treatment [7]. Beyond targeted therapies, various ICI have been tested as novel first-line treatments for mRCC. In recent clinical trials, ICI-based combination therapies including nivolumab plus ipilimumab, atezolizumab plus 
bevacizumab, pembrolizumab plus axitinib, and avelumab plus axitinib exhibited significant benefits in terms of overall survival (OS) and/or progression-free survival (PFS) benefit for $\mathrm{mRCC}$ compared with sunitinib as a standard first-line treatment for mRCC [8-11]. Moreover, updating result of the KEYNOTE-426 trial was recently reported [12]. However, there are scant direct comparative data between these agents to inform optimal treatment decisions and guideline recommendations. Therefore, a systematic review was conducted in all clinical trials assessing first-line systemic therapy of mRCC with sunitinib as the control arm, and network meta-analyses were also conducted to indirectly compare the efficacy and safety of the first-line treatment options.

\section{Materials and methods}

The protocol has been registered in the International Prospective Register of Systematic Reviews database (PROSPERO: CRD42020170483).

\section{Search strategy}

The systematic review and network meta-analysis of phase III randomized controlled trials (RCT) comparing at least two first-line systemic therapies for mRCC (with sunitinib monotherapy as the control arm) were conducted according to the preferred reporting items for systematic reviews and meta-analyses (PRISMA) extension statement for network meta-analysis [13]. A completed PRISMA 2009 checklist was used to describe the methodology of our study (Supplementary Table 1). The PubMed, Web of Science, and Scopus databases were searched to identify reports published until June 2020 in first-line systemic therapy for mRCC. The following keywords were used in our search strategy: (renal cell carcinoma OR renal cell cancer OR kidney carcinoma OR kidney cancer) AND (metastatic OR advanced) AND (Randomized). The primary outcome of interest was OS and PFS, and the secondary outcomes were objective response, and adverse events (AEs). Initial screening was performed independently by two investigators based on the titles and abstracts of the article to identify ineligible reports. Reasons for exclusions were noted. Potentially relevant reports were subjected to a full-text review, and the relevance of the reports was confirmed after the data extraction process. Disagreements were resolved via consensus with a separate committee of investigators.

\section{Inclusion and exclusion criteria}

Studies were included if they investigated metastatic clearcell RCC patients (Patients) who had undergone systemic therapy as first-line treatment (Intervention) compared with those treated with sunitinib as first-line treatment (Comparison) to assess the differential effects on PFS, OS, objective response, and AEs (Outcome) in phase III randomized studies only. We excluded observational studies, reviews, letters, editorials, replies from authors, case reports, and articles not published in English. In cases of multiple publications on the same cohort, either the higher quality or the most recent publication was selected. References of all papers included were scanned for additional studies of interest. As the focus of this study was the first-line efficacy of these agents in patients who had no history of systemic therapy, studies involving patients who had a history of systemic therapy or studies in which this subset could not be excluded from the overall cohort were excluded from this analysis. As TKIs are widely accepted as the standard of care, studies were excluded if they included interferon or placebo as the control arms. Studies were included only if they involved patients who received sunitinib $50 \mathrm{mg}$ as the control arm.

\section{Data extraction}

Two investigators independently extracted the following information from the included articles: first author's name, publication year, period of patient recruitment, number of patients, treatment dosage, age, sex, study design, risk group, component of RCC, oncologic outcomes, and AE outcomes. Subsequently, the hazard ratios (HR) and 95\% confidence intervals (CI) associated with PFS and OS, objective response rate, and $\mathrm{AE}$ rate were retrieved. All HRs were derived from Cox models. All discrepancies regarding data extraction were resolved by consensus with the committee of investigators.

\section{Risk of bias assessment}

The "risk-of-bias" (RoB) evaluation of each study was assessed according to The Cochrane Collaboration's tool for assessing risk of bias [14]. This tool assesses selection bias (random sequence generation and allocation concealment), performance bias, detection bias, attrition bias, reporting bias, and other sources of bias (Supplementary Fig 1). The RoB of each study was assessed independently by two authors. Disagreements were resolved by consultation with the coauthors.

\section{Statistical analyses}

PFS was defined as the time from randomization to the first radiographic progression or death due to any cause. Objective response was defined as the proportion of enrolled and randomly assigned patients who achieved the best response of complete response (CR) or partial response based on investigator assessment. For each outcome, we conducted 
network meta-analysis using random and fixed-effect models with a Bayesian approach for the direct and indirect treatment comparisons with sunitinib as the common comparator arm $[15,16]$. In the assessment for PFS and OS, contrastbased analyses were applied with estimated differences in the $\log \mathrm{HR}$ and the standard error calculated from the published HR and CI [17]. The relative treatment effects were presented as HR and 95\% credible interval (CrI) [15]. With regard to PFS and OS, subgroup analyses were conducted among: intermediate/poor-risk disease and favorable-risk disease defined according to the Memorial Sloan Kettering Cancer Center or International mRCC Database Consortium risk categorization $[18,19]$. For the assessment of the objective response and AEs, arm-based analyses were performed to estimate ORs of the objective response and AEs (and 95\% CrI) from the available raw data presented in the selected manuscripts [15]. We also estimated the relative ranking of the different treatments for each outcome using the $P$ score, which can be considered a frequentist analog to the surface under the cumulative ranking curves [20,21]. Network plots were utilized to illustrate the connectivity of the treatment networks in terms of OS, PFS, and AEs. Heterogeneity was assessed using $I^{2}$ when more than one trial was available for a given comparison. All statistical analyses were performed using R and Stata/MP 14.2 (Stata Corp., College Station, TX); statistical significance was set at $P<0.05$.

\section{Results}

\section{Study selection and characteristics}

Our initial search identified 4116 publications, and after the elimination of duplicates, a total of 3667 publications were available. A total of 3611 articles were excluded after screening the titles and abstracts, and a full-text review was performed for 56 articles (Supplementary Figure S1). Based on the selection criteria, we identified 6 articles comprising 5297 patients for the systematic review and network metaanalysis [8-12, 22-24]. Extracted data from the six studies are outlined in Tables 1, S2. All these studies were published between 2013 and 2019 and included 2568 [male: 1912 (74.5\%); age range: $61-62$ years] patients treated with sunitinib and 2639 [male: 1895 (71.8\%); age range: 60-62 years] patients treated with other systemic agents.

\section{Network meta-analysis}

The networks of eligible comparisons were graphically represented in network plots in terms of PFS (Supplementary Fig. 2a), OS (Supplementary Fig. 2b), and high-grade AEs (Supplementary Fig. 2c).

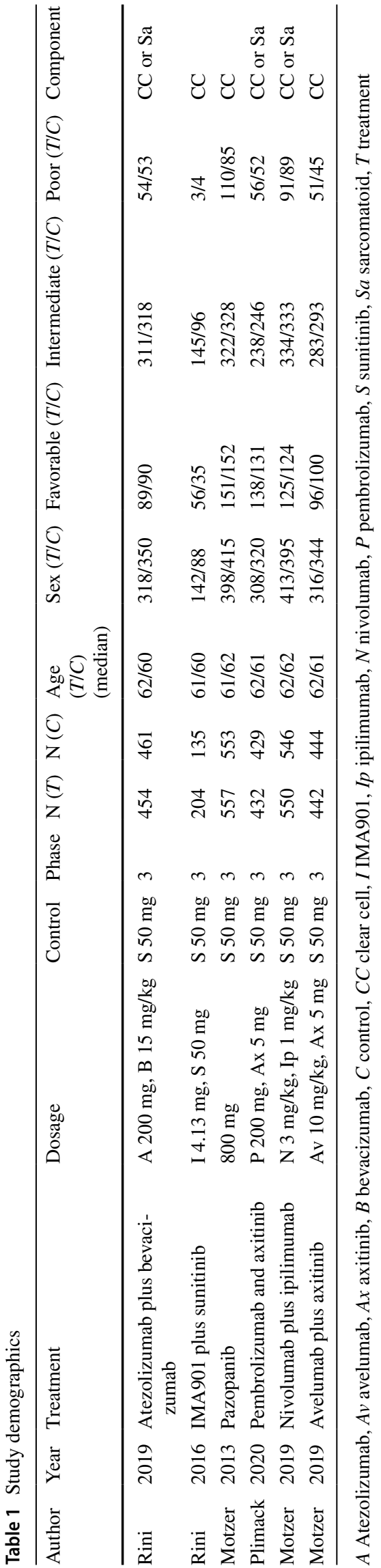




\section{Progression-free survival}

A network meta-analysis of seven different agents was conducted for the primary outcome of PFS. Compared with sunitinib, avelumab plus axitinib and pembrolizumab plus axitinib resulted in significantly improved PFS (HR $0.85,95 \%$ CrI 0.74-0.98 and HR 0.86, 95\% CrI 0.76-0.97, respectively) (Fig. 1a). Based on analysis of the treatment ranking, avelumab plus axitinib had the highest likelihood of providing the maximal PFS ( $P$ score: 0.8255 ) (Table 2). Pembrolizumab plus axitinib was likely to be similarly deemed as the preferred treatment choice $(P$ score: 0.8022).

\section{Overall survival}

A network meta-analysis of seven different agents was conducted for the primary outcome of OS. Compared with sunitinib, nivolumab plus ipilimumab, and pembrolizumab plus axitinib resulted in significantly improved OS (HR 0.86, 95\% CrI 0.75-0.99 and HR 0.85, 95\% CrI 0.73-0.98, respectively) (Fig. 1b). Based on analysis of the treatment ranking, pembrolizumab plus axitinib had the highest likelihood of providing the maximal OS ( $P$ score: 0.8052$)$ (Table 2$)$. Nivolumab plus ipilimumab was likely to be similarly deemed as the preferred treatment choice ( $P$ score: 0.7625$)$.

\section{Adverse events}

Rates of grade $3 \geqq$ AEs were examined as a measure of toxicity of treatment. A network meta-analysis of six different agents was conducted for the outcome of serious AEs. Compared with sunitinib, nivolumab plus ipilimumab (OR $0.50,95 \%$ CrI 0.39-0.64) was associated with a significantly lower likelihood of toxicity (Fig. 1c). Based on analysis of the treatment ranking, it was highly likely that nivolumab plus ipilimumab had the lowest rate of serious AEs ( $P$ score: 0.9999) (Table 2).

\section{Objective response}

A network meta-analysis of six agents was performed for the outcome of objective response rates. Compared with sunitinib, avelumab plus axitinib and nivolumab plus ipilimumab and pazopanib, and pembrolizumab plus axitinib resulted in significantly higher objective response rates (Fig. 2a). Based on analysis of the treatment ranking, it was highly likely that avelumab plus axitinib had the highest objective response rate ( $P$ score: 0.9855$)$, closely followed by pembrolizumab plus axitinib ( $P$ score: 0.8132 ) (Supplementary Table 2 ).

\section{Complete response}

A network meta-analysis of six agents was conducted for the outcome of CR rates. Compared with sunitinib, atezolizumab plus bevacizumab and nivolumab plus ipilimumab, and pembrolizumab plus axitinib resulted in significantly higher CR rates (Fig. 2b). Based on analysis of the treatment ranking, it was highly likely that Nivolumab plus ipilimub had the highest $C R$ rate ( $P$ score: 0.9742 ), followed by pembrolizumab plus axitinib ( $P$ score: 0.6998$)$ (Supplementary Table 2).

\section{Intermediate/poor-risk subgroup}

Based on analysis of the treatment ranking, in patients with intermediate/poor-risk mRCC, pembrolizumab plus axitinib had the highest likelihood of providing the maximal OS $(P$ score: 0.8220 ), closely followed by nivolumab plus ipilimumab ( $P$ score: 0.7677). Based on Bayesian analysis and analysis of the treatment ranking, avelumab plus axitinib had the highest likelihood of providing the maximal PFS ( $P$ score: 0.7582$)$, closely followed by pembrolizumab plus axitinib ( $P$ score: 0.7293 ) (Supplementary Fig. 3 and Supplementary Table 3$)$.

\section{Favorable-risk subgroup}

Based on analysis of the treatment ranking, in patients with favorable-risk mRCC, IMA901 plus sunitinib had the highest likelihood of providing the maximal OS ( $P$ score: 0.6136). Based on Bayesian analysis and analysis of the treatment ranking, avelumab plus axitinib had the highest likelihood of providing the maximal PFS ( $P$ score: 0.8480 ) (Supplementary Fig. 3 and Supplementary Table 3).

\section{Discussion}

A systematic review was conducted on systemic therapy agents that have been evaluated in RCTs for patients with $\mathrm{mRCC}$ in comparison with sunitinib in the first-line setting; we also performed a network meta-analysis and indirectly compared clinically relevant first-line treatment options. This approach generated several important findings. First, pembrolizumab plus axitinib was probably the best treatment option with regard to survival, and statistically more effective than most available treatments. Second, avelumab plus axitinib and nivolumab plus ipilimumab were probably the second best options with regard to survival. Third, nivolumab plus ipilimumab was the best tolerated of all the agents evaluated. The ICI-based combination treatments 
A

Treatment

Comparison: other vs 'Sunitinib'

Atezolizumab plus bevacizumab

Avelumab plus axitinib

IMA901 plus sunitinib

Nivolumab plus ipilimumab

Pazopanib

Pembrolizumab plus axitinib

Sunitinib

(Random Effects Model) HR $\quad 95 \%-\mathrm{Cl}$

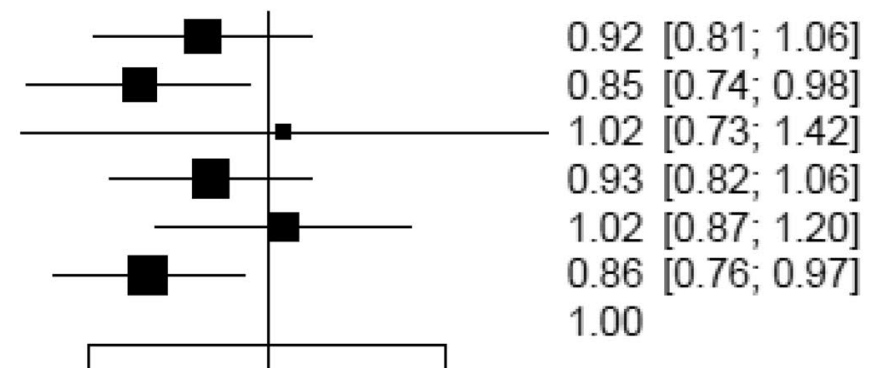

$\begin{array}{lll}0.8 & 1 & 1.25\end{array}$

B

Treatment

Atezolizumab plus bevacizumab

Avelumab plus axitinib

IMA901 plus sunitinib

Nivolumab plus ipilimumab

Pazopanib

Pembrolizumab plus axitinib

Sunitinib
Comparison: other vs 'Sunitinib' (Random Effects Model)

$\mathrm{HR} \quad 95 \%-\mathrm{Cl}$

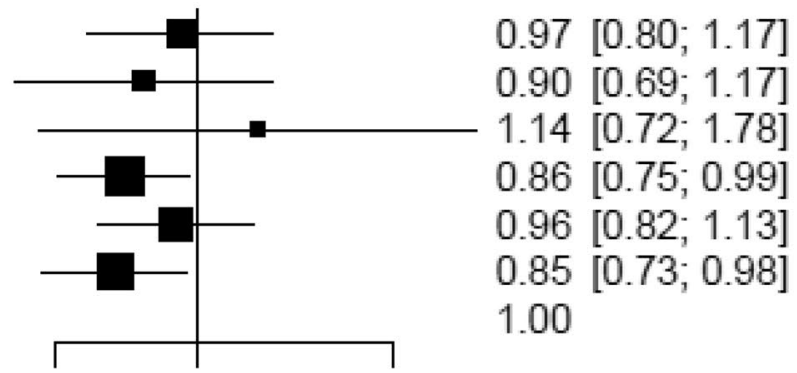

$\begin{array}{lll}0.75 & 1 & 1.5\end{array}$
C

Treatment

Avelumab plus axitinib IMA901 plus sunitinib Nivolumab plus ipilimumab Pazopanib

Pembrolizumab plus axitinib Sunitinib
Comparison: other vs 'Sunitinib'

(Random Effects Model) OR $\quad 95 \%-\mathrm{Cl}$

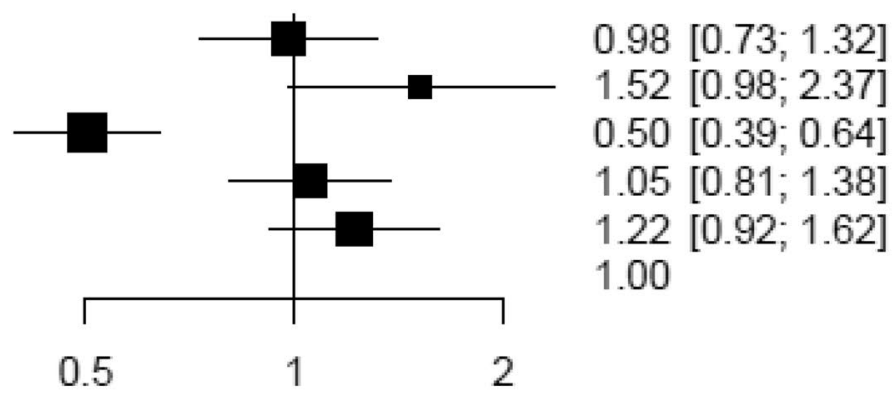

Fig. 1 Forest plots showing the association of systemic therapy in metastatic renal cell carcinoma. a progression-free survival (PFS), b overall survival (OS), c adverse event (AE)

(nivolumab plus ipilimumab, pembrolizumab plus axitinib, and avelumab plus axitinib) were associated with fewer or similar high-grade AEs than sunitinib.
These developments are of particular interest, as previous network meta-analyses did not include recently reported data and/or analyzed heterogeneous populations 
Table 2 Analysis of the treatment ranking

\begin{tabular}{|c|c|c|}
\hline Treatment & $P$ score $($ fixed) & $P$ score (random) \\
\hline \multicolumn{3}{|l|}{ Progression free survival } \\
\hline Avelumab plus axitinib & 0.8255 & 0.8255 \\
\hline Pembrolizumab plus axitinib & 0.8002 & 0.8002 \\
\hline $\begin{array}{l}\text { Atezolizumab plus bevaci- } \\
\text { zumab }\end{array}$ & 0.5679 & 0.5679 \\
\hline Nivolumab plus ipilimumab & 0.5316 & 0.5316 \\
\hline IMA901 plus sunitinib & 0.3122 & 0.3122 \\
\hline Sunitinb & 0.2377 & 0.2377 \\
\hline Pazopanib & 0.2249 & 0.2249 \\
\hline \multicolumn{3}{|l|}{ Overall survival } \\
\hline Pembrolizumab plus axitinib & 0.8052 & 0.8052 \\
\hline Nivolumab plus ipilimumab & 0.7625 & 0.7625 \\
\hline Avelumab plus axitinib & 0.6141 & 0.6141 \\
\hline Pazopanib & 0.433 & 0.433 \\
\hline $\begin{array}{l}\text { Atezolizumab plus bevaci- } \\
\text { zumab }\end{array}$ & 0.4094 & 0.4094 \\
\hline Sunitinb & 0.2721 & 0.2721 \\
\hline IMA901 plus sunitinib & 0.2038 & 0.2038 \\
\hline \multicolumn{3}{|l|}{ Adverse events } \\
\hline Nivolumab plus ipilimumab & 0.9999 & 0.9999 \\
\hline Sunitinb & 0.5985 & 0.5985 \\
\hline Avelumab plus axitinib & 0.5944 & 0.5944 \\
\hline Pazopanib & 0.4817 & 0.4817 \\
\hline Pembrolizumab plus axitinib & 0.2517 & 0.2517 \\
\hline IMA901 plus sunitinib & 0.0737 & 0.0737 \\
\hline
\end{tabular}

$[25,26]$. Therefore, we focused only on phase-3 studies which included sunitinib as the control arm and included recently published data, such as updated results from the KEYNOTE-426 trial [12]. As a result, this design may be more reasonable compared with an already published paper by Hahn et al. [25]. In addition, this network meta-analysis included AE outcomes. This is of greater relevance to clinical practice than the recent study by Monteiro et al. [26]. On these points, current paper may more readily facilitate individualized treatment selection.

In this meta-analysis, pembrolizumab plus axitinib, a combination of an anti-programmed death 1 (PD-1) monoclonal antibody and VEGF receptor (VEGFR) TKI, appeared to be the best therapeutic option based on its benefit for OS and PFS. Blockade of immune checkpoints, such as cytotoxic T-lymphocyte-associated antigen 4 (CTLA-4) and PD-1, that are negative regulators which inhibit T-cell proliferation and activity, could result in tumor eradication through reactivation and enhancement of the internal T-cell response [27]; moreover, VEGF inhibition has been shown to suppress angiogenesis as well as increase the recruitment and tumor infiltration of T cells [28, 29]. Indeed, it has been shown in mouse models that simultaneous inhibition of the
VEGF and PD-1 pathways not only reduced tumor neovascularization and upregulation of pro-inflammatory cytokines but also inhibited tumor growth [30,31]. Thus, the finding that the combination of ICI and VEGF axis inhibitors could play a key role in the treatment of mRCC led to a large number of clinical trials to test such combinations. Our pooled analysis of the effects of these combinations revealed that pembrolizumab plus axitinib represented the best treatment with regard to OS and PFS, despite major concerns over toxicity from previous studies combining PD-1 inhibitors nivolumab and pembrolizumab with sunitinib or pazopanib $[32,33]$. However, in this analysis, pembrolizumab plus axitinib resulted in similar high-grade AEs to those with sunitinib; this may be because axitinib is a highly selective inhibitor of VEGFR, while sunitinib and pazopanib have a broader range of targets [34].

The use of nivolumab in combination with ipilimumab, a dual checkpoint inhibitor with selective affinity for immune cells that express PD- 1 and CTLA- 4 molecules, produced impressive results in mRCC patients $[8,22]$. While nivolumab plus ipilimumab may not be as effective as pembrolizumab plus axitinib according to our network meta-analysis, this combination was superior to sunitinib, both with regard to PFS/OS and its safety profile, as it was associated with fewer serious AEs. Thus, nivolumab plus ipilimumab appears to provide the most favorable-risk/benefit treatment.

Despite the comprehensive nature of the systematic review undertaken, there are some limitations that need to be considered. First, although indirect treatment comparison analyses have been used and validated for comparing outcomes from RCTs, this approach falls short of a headto-head treatment comparison. Thus, direct well-designed comparative trials are required to validate the findings of this study. Second, this network meta-analysis was based on the reporting quality of the trials we reviewed and may have been affected by several types of bias, thus limiting the validity of the overall findings. Third, the patient characteristics may have differed significantly between the studies, limiting the comparability of the trials evaluated. Moreover, the OS benefits of the treatments were not evaluated in some of the trials that assessed PFS as the primary endpoint, which did not allow the comprehensive evaluations of OS benefits of all existing treatments. While not being an individual patient data meta-analysis, this study demonstrated no major differences in the patient characteristics, suggesting that some confounding factors, such as prognostic risk categories and PD-L1 status, may have influenced the systemic treatment benefit; this was not ascertainable at the individual patient level. Moreover, because of the limitations of published data, performing a meta-analysis of adjusted effect estimates proved to be impossible. Fourth, the doses and methods 
A

Treatment

Atezolizumab plus bevacizumab

Avelumab plus axitinib

Nivolumab plus ipilimumab

Pazopanib

Pembrolizumab plus axitinib

Sunitinib
Comparison: other vs 'Sunitinib'

(Random Effects Model) OR $\quad 95 \%-\mathrm{Cl}$

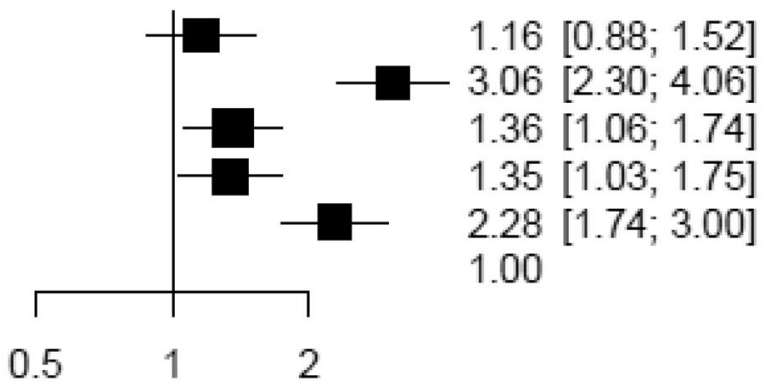

B

Treatment

Atezolizumab plus bevacizumab

Avelumab plus axitinib

Nivolumab plus ipilimumab

Pazopanib

Pembrolizumab plus axitinib

Sunitinib

\section{Comparison: other vs 'Sunitinib'}

(Random Effects Model) OR $\quad 95 \%-\mathrm{Cl}$

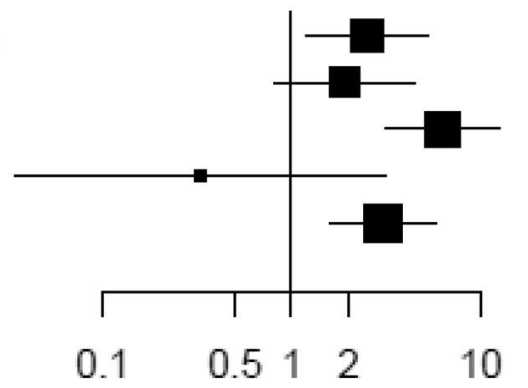

$2.52[1.19 ; 5.33]$

$1.91[0.80 ; 4.56]$

$6.32[3.19 ; 12.50]$

$0.33[0.03 ; 3.18]$

$3.09[1.62 ; 5.88]$

1.00

Fig. 2 Forest plots showing the association of systemic therapy in metastatic renal cell carcinoma. a Objective response, $\mathbf{b}$ complete response

of administration of each systemic treatment in the studies included may differ from those in real-world clinical practice, and therefore, the efficacy and toxicity may have differed according to the dose and method of administration. Most notably, while individualized dose adjustment of sunitinib has been shown to improve its efficacy and tolerability, the studies included in this analysis employed the standard dose regimen only $[35,36]$. Fifth, some of the treatments evaluated here are least likely to reach the clinic and/or remain of only relative interest (e.g., sunitinib + IMA901). Finally, differences in subsequent therapies received across the treatment arms in the trials evaluated may have possibly influenced the OS results. In addition, the OS data from some trials were immature; thus, the study outcomes could change in their final analysis. Nevertheless, the current network meta-analysis suggests that pembrolizumab plus axitinib appears to be the best option in the first-line setting. This could help improve clinical decision making, while the present metaanalysis is not intended to replace the need for head-tohead clinical trials of contemporary first-line therapies.

\section{Conclusion}

In this systematic review and network meta-analysis of first-line systemic therapies for patients with $\mathrm{mRCC}$, based on an indirect comparison of data from phase-3 clinical trials, pembrolizumab plus axitinib was identified as having a high likelihood of providing the maximum PFS and OS benefits. In addition, nivolumab plus ipilimumab appeared to have the most favorable tradeoff between efficacy and tolerability. These findings may provide guidance to patients and clinicians for treatment decisions, when considered with other aspects that drive personalized medicine strategies for mRCC.

Acknowledgements Open access funding provided by Medical University of Vienna.

Author contributions Project development: KM, PIK, SE, SFS. Data collection: KM, HM, NM. Data analysis: KM, HM. Manuscript writing/editing: KM, HM, NM, PIK, SL, MS, BP, SE, SFS. 
Funding This research did not receive any specific Grant from funding agencies in the public, commercial, or not-for-profit sectors.

\section{Compliance with ethical standards}

Conflict of interest None of the authors have conflicts of interest to disclose.

Open Access This article is licensed under a Creative Commons Attribution 4.0 International License, which permits use, sharing, adaptation, distribution and reproduction in any medium or format, as long as you give appropriate credit to the original author(s) and the source, provide a link to the Creative Commons licence, and indicate if changes were made. The images or other third party material in this article are included in the article's Creative Commons licence, unless indicated otherwise in a credit line to the material. If material is not included in the article's Creative Commons licence and your intended use is not permitted by statutory regulation or exceeds the permitted use, you will need to obtain permission directly from the copyright holder. To view a copy of this licence, visit http://creativecommons.org/licenses/by/4.0/.

\section{References}

1. Stitzlein L, Rao P, Dudley R (2019) Emerging oral VEGF inhibitors for the treatment of renal cell carcinoma. Expert Opin Investig Drugs 28:121-130. https://doi.org/10.1080/13543784.2019.15592 96

2. Flanigan RC, Campbell SC, Clark JI, Picken MM (2003) Metastatic renal cell carcinoma. Curr Treat Options Oncol 4:385-390. https://doi.org/10.1007/s11864-003-0039-2

3. Gill DM, Hahn AW, Hale P, Maughan BL (2018) Overview of current and future first-line systemic therapy for metastatic clear cell renal cell carcinoma. Curr Treat Options Oncol 19:6. https:// doi.org/10.1007/s11864-018-0517-1

4. Posadas EM, Limvorasak S, Figlin RA (2017) Targeted therapies for renal cell carcinoma. Nat Rev Nephrol 13:496-511. https:// doi.org/10.1038/nrneph.2017.82

5. Choueiri TK, Motzer RJ (2017) Systemic therapy for metastatic renal-cell carcinoma. N Engl J Med 376:354-366. https://doi. org/10.1056/NEJMra1601333

6. McDermott DF, Regan MM, Clark JI et al (2005) Randomized phase III trial of high-dose interleukin-2 versus subcutaneous interleukin-2 and interferon in patients with metastatic renal cell carcinoma. J Clin Oncol 23:133-141. https://doi.org/10.1200/ jco.2005.03.206

7. Heng DY, Xie W, Regan MM et al (2009) Prognostic factors for overall survival in patients with metastatic renal cell carcinoma treated with vascular endothelial growth factor-targeted agents: results from a large, multicenter study. J Clin Oncol 27:57945799. https://doi.org/10.1200/jco.2008.21.4809

8. Motzer RJ, Tannir NM, McDermott DF et al (2018) Nivolumab plus ipilimumab versus sunitinib in advanced renal-cell carcinoma. N Engl J Med 378:1277-1290. https://doi.org/10.1056/ NEJMoa1712126

9. Rini BI, Powles T, Atkins MB et al (2019) Atezolizumab plus bevacizumab versus sunitinib in patients with previously untreated metastatic renal cell carcinoma (IMmotion151): a multicentre, open-label, phase 3, randomised controlled trial. Lancet 393:2404-2415. https://doi.org/10.1016/s0140-6736(19)30723-8
10. Rini BI, Plimack ER, Stus V et al (2019) Pembrolizumab plus axitinib versus sunitinib for advanced renal-cell carcinoma. N Engl J Med 380:1116-1127. https://doi.org/10.1056/NEJMoa1816714

11. Motzer RJ, Penkov K, Haanen J et al (2019) Avelumab plus axitinib versus sunitinib for advanced renal-cell carcinoma. N Engl J Med 380:1103-1115. https://doi.org/10.1056/NEJMoa1816047

12. Plimack ER, Rini BI, Stus V et al (2020) Pembrolizumab plus axitinib versus sunitinib as first-line therapy for advanced renal cell carcinoma (RCC): updated analysis of KEYNOTE-426. J Clin Oncol 38:5001. https://doi.org/10.1200/JCO.2020.38.15_suppl .5001

13. Hutton B, Salanti G, Caldwell DM et al (2015) The PRISMA extension statement for reporting of systematic reviews incorporating network meta-analyses of health care interventions: checklist and explanations. Ann Intern Med 162:777-784. https://doi. org $/ 10.7326 / \mathrm{m} 14-2385$

14. Higgins JP, Altman DG, Gotzsche PC et al (2011) The Cochrane Collaboration's tool for assessing risk of bias in randomised trials. BMJ 343:d5928. https://doi.org/10.1136/bmj.d5928

15. van Valkenhoef G, Lu G, de Brock B, Hillege H, Ades AE, Welton NJ (2012) Automating network meta-analysis. Res Synth Methods 3:285-299. https://doi.org/10.1002/jrsm.1054

16. Dias S, Welton NJ, Sutton AJ, Ades AE (2014) NICE decision support unit technical support documents. In: NICE DSU technical support document 2: a generalised linear modelling framework for pairwise and network meta-analysis of randomised controlled trials

17. Woods BS, Hawkins N, Scott DA (2010) Network meta-analysis on the log-hazard scale, combining count and hazard ratio statistics accounting for multi-arm trials: a tutorial. BMC Med Res Methodol 10:54. https://doi.org/10.1186/1471-2288-10-54

18. Motzer RJ, Mazumdar M, Bacik J, Berg W, Amsterdam A, Ferrara J (1999) Survival and prognostic stratification of 670 patients with advanced renal cell carcinoma. J Clin Oncol 17:2530-2540. https ://doi.org/10.1200/jco.1999.17.8.2530

19. Ko JJ, Xie W, Kroeger N et al (2015) the international metastatic renal cell carcinoma database consortium model as a prognostic tool in patients with metastatic renal cell carcinoma previously treated with first-line targeted therapy: a population-based study. Lancet Oncol 16:293-300. https://doi.org/10.1016/s1470 $-2045(14) 71222-7$

20. Rucker G, Schwarzer G (2015) Ranking treatments in frequentist network meta-analysis works without resampling methods. BMC Med Res Methodol 15:58. https://doi.org/10.1186/s1287 4-015-0060-8

21. Salanti G, Ades AE, Ioannidis JP (2011) Graphical methods and numerical summaries for presenting results from multiple-treatment meta-analysis: an overview and tutorial. J Clin Epidemiol 64:163-171. https://doi.org/10.1016/j.jclinepi.2010.03.016

22. Motzer RJ, Rini BI, McDermott DF et al (2019) Nivolumab plus ipilimumab versus sunitinib in first-line treatment for advanced renal cell carcinoma: extended follow-up of efficacy and safety results from a randomised, controlled, phase 3 trial. Lancet Oncol 20:1370-1385. https://doi.org/10.1016/s1470-2045(19)30413-9

23. Rini BI, Stenzl A, Zdrojowy R et al (2016) IMA901, a multipeptide cancer vaccine, plus sunitinib versus sunitinib alone, as first-line therapy for advanced or metastatic renal cell carcinoma (IMPRINT): a multicentre, open-label, randomised, controlled, phase 3 trial. Lancet Oncol 17:1599-1611. https://doi. org/10.1016/s1470-2045(16)30408-9

24. Motzer RJ, Hutson TE, Cella D et al (2013) Pazopanib versus sunitinib in metastatic renal-cell carcinoma. N Engl J Med 369:722-731. https://doi.org/10.1056/NEJMoa1303989

25. Hahn AW, Klaassen Z, Agarwal N, Haaland B, Esther J, Ye XY, Wang X, Pal SK, Wallis CJD (2019) First-line treatment of metastatic renal cell carcinoma: a systematic review and 
network meta-analysis. Eur Urol Oncol 2:708-715. https://doi. org/10.1016/j.euo.2019.09.002

26. Monteiro FSM, Soares A, Debiasi M et al (2020) First-line treatment of metastatic renal cell carcinoma in the immuno-oncology era: systematic review and network meta-analysis. Clin Genitourin Cancer. https://doi.org/10.1016/j.clgc.2020.02.012

27. Carlo MI, Voss MH, Motzer RJ (2016) Checkpoint inhibitors and other novel immunotherapies for advanced renal cell carcinoma. Nat Rev Urol 13:420-431. https://doi.org/10.1038/nruro 1.2016 .103

28. Manning EA, Ullman JG, Leatherman JM, Asquith JM, Hansen TR, Armstrong TD, Hicklin DJ, Jaffee EM, Emens LA (2007) A vascular endothelial growth factor receptor-2 inhibitor enhances antitumor immunity through an immune-based mechanism. Clin Cancer Res 13:3951-3959. https://doi.org/10.1158/1078-0432. ccr-07-0374

29. Shrimali RK, Yu Z, Theoret MR, Chinnasamy D, Restifo NP, Rosenberg SA (2010) Antiangiogenic agents can increase lymphocyte infiltration into tumor and enhance the effectiveness of adoptive immunotherapy of cancer. Cancer Res 70:6171-6180. https://doi.org/10.1158/0008-5472.can-10-0153

30. Turajlic S, Swanton C, Boshoff C (2018) Kidney cancer: the next decade. J Exp Med 215:2477-2479. https://doi.org/10.1084/ jem.20181617

31. Yasuda S, Sho M, Yamato I, Yoshiji H, Wakatsuki K, Nishiwada S, Yagita H, Nakajima Y (2013) Simultaneous blockade of programmed death 1 and vascular endothelial growth factor receptor 2 (VEGFR2) induces synergistic anti-tumour effect in vivo. Clin Exp Immunol 172:500-506. https://doi.org/10.1111/cei.12069

32. Amin A, Plimack ER, Ernstoff MS et al (2018) Safety and efficacy of nivolumab in combination with sunitinib or pazopanib in advanced or metastatic renal cell carcinoma: the CheckMate 016 study. J Immunother Cancer 6:109. https://doi.org/10.1186/s4042 5-018-0420-0

33. Chowdhury S, McDermott DF, Voss MH, Hawkins RE, Aimone P, Voi M, Isabelle N, Wu Y, Infante JR (2017) A phase I/II study to assess the safety and efficacy of pazopanib (PAZ) and pembrolizumab (PEM) in patients (pts) with advanced renal cell carcinoma (aRCC). J Clin Oncol 35:4506. https://doi.org/10.1200/ JCO.2017.35.15_suppl.4506

34. Atkins MB, Plimack ER, Puzanov I et al (2018) Axitinib in combination with pembrolizumab in patients with advanced renal cell cancer: a non-randomised, open-label, dose-finding, and doseexpansion phase 1b trial. Lancet Oncol 19:405-415. https://doi. org/10.1016/s1470-2045(18)30081-0

35. Kalra S, Rini BI, Jonasch E (2015) Alternate sunitinib schedules in patients with metastatic renal cell carcinoma. Ann Oncol 26:1300-1304. https://doi.org/10.1093/annonc/mdv030

36. Basappa NS, Lalani A-KA, Li H et al (2017) Individualized treatment with sunitinib versus standard dosing with sunitinib or pazopanib in patients with metastatic renal cell carcinoma (mRCC): results from the Canadian kidney cancer information system (CKCis). J Clin Oncol 35:468. https://doi.org/10.1200/ JCO.2017.35.6_suppl.468

Publisher's Note Springer Nature remains neutral with regard to jurisdictional claims in published maps and institutional affiliations.

\section{Affiliations}

\section{Keiichiro Mori ${ }^{1,2} \oplus$ - Hadi Mostafaei ${ }^{1,3} \cdot$ Noriyoshi Miura $^{1,4} \cdot$ Pierre I. Karakiewicz ${ }^{5}$ Stefano Luzzago ${ }^{5,6} \cdot$ Manuela Schmidinger ${ }^{7}$. Andreas Bruchbacher ${ }^{1,7}$. Benjamin Pradere ${ }^{1,8,16} \cdot$ Shin Egawa ${ }^{2}$. Shahrokh F. Shariat ${ }^{1,9,10,11,12,13,14,15}$}

1 Department of Urology, Medical University of Vienna, Währinger Gürtel 18-20, 1090 Vienna, Austria

2 Department of Urology, The Jikei University School of Medicine, Tokyo, Japan

3 Research Center for Evidence Based Medicine, Tabriz University of Medical Sciences, Tabriz, Iran

4 Department of Urology, Ehime University Graduate School of Medicine, Ehime, Japan

5 Cancer Prognostics and Health Outcomes Unit, University of Montreal Health Centre, Montreal, Canada

6 Department of Urology, European Institute of Oncology, IRCCS, Milan, Italy

7 Clinical Division of Oncology, Department of Medicine I and Comprehensive Cancer Center, Medical University of Vienna, Vienna, Austria

8 Department of Urology, University Hospital of Tours, Tours, France
9 Division of Urology, Department of Special Surgery, The University of Jordan, Amman, Jordan

10 Department of Urology, Weill Cornell Medical College, New York, NY, USA

11 Department of Urology, University of Texas Southwestern, Dallas, TX, USA

12 Karl Landsteiner Institute of Urology and Andrology, Vienna, Austria

13 Department of Urology, Second Faculty of Medicine, Charles University, Prague, Czech Republic

14 Institute for Urology and Reproductive Health, I.M. Sechenov First Moscow State Medical University, Moscow, Russia

15 European Association of Urology Research Foundation, Arnhem, Netherlands

16 Université François Rabelais de Tours, PRES Centre Val de Loire, Tours, France 